\title{
Testing a Model of Poliovirus Cell-Entry via Virus-Antibody Interactions
}

\author{
J. Lin ${ }^{*}$, N. Cheng ${ }^{* *}$, A.C. Steven ${ }^{* *}$, J. M. Hogle ${ }^{* * *}$, and D. M. Belnap ${ }^{* * *}$ \\ *Department of Chemistry and Biochemistry, Brigham Young University, Provo, UT 84602 \\ **Laboratory of Structural Biology Research, National Institute of Arthritis, Musculoskeletal \\ and Skin Diseases, National Institutes of Health, Bethesda, MD 20892 \\ ***Department of Biological Chemistry and Molecular Pharmacology, Harvard Medical School, \\ Boston, MA 02115
}

Poliovirus is an excellent model system for understanding the cell-entry mechanism of nonenveloped viruses [1]. The virion (sedimentation coefficient $=160 \mathrm{~S}$ ) interacts with its receptor and changes into an intermediate (135S) form. The transition expels two membrane-binding, protein entities (VP4 and the N-terminus of VP1), which are thought to allow the 135S particle to interact with the cell membrane and facilitate viral penetration. After releasing RNA into the infected cell, the empty poliovirus sediments at $80 \mathrm{~S}$.

Two previous studies [2,3] used cryogenic electron microscopy (cryo-EM) to determine threedimensional structures of all three intermediate particles. After determining the 3D structures, poliovirus crystal structure coordinates [4] were fitted into the three-dimensional cryo-EM reconstructions to make a pseudo-atomic model of the transitions. The model of the intermediate particle is that the N-terminus of VP1 extends through a small opening between adjacent subunits, an $\alpha$-helix is formed that lies against the particle surface near the exit site, and the Nterminal 31 residues are mobile or disordered. With some exceptions, the $80 \mathrm{~S}$ particle resembles the $135 \mathrm{~S}$ particle.

We studied the 160S-to-135S transition via different monoclonal antibodies to VP1. Fabs, the antibody-binding portion of the antibody, were mixed with virus particles and cryo-EM specimens of the complexes were prepared and imaged. From the images, we computed 3D reconstructions of the complexes (Fig. 1). One antibody, to a constantly exposed portion of VP1, is found in that location. Another antibody to the N-terminal 31 residues is found near the predicted site in the $135 \mathrm{~S}$ and $80 \mathrm{~S}$ particles. These results are consistent with the positions of these portions of VP1 as modeled by Belnap et al. [2] and Bubeck et al.[3].

\section{References}

[1] J.M. Hogle, Ann. Rev. Microbiol. 56 (2002) 677.

[2] D.M. Belnap et al., J. Virol. 74 (2000) 1342.

[3] D. Bubeck et al., J. Virol. 79 (2005) 7745.

[4] S.T. Miller et al., J. Mol. Biol. 307 (2001) 499. 

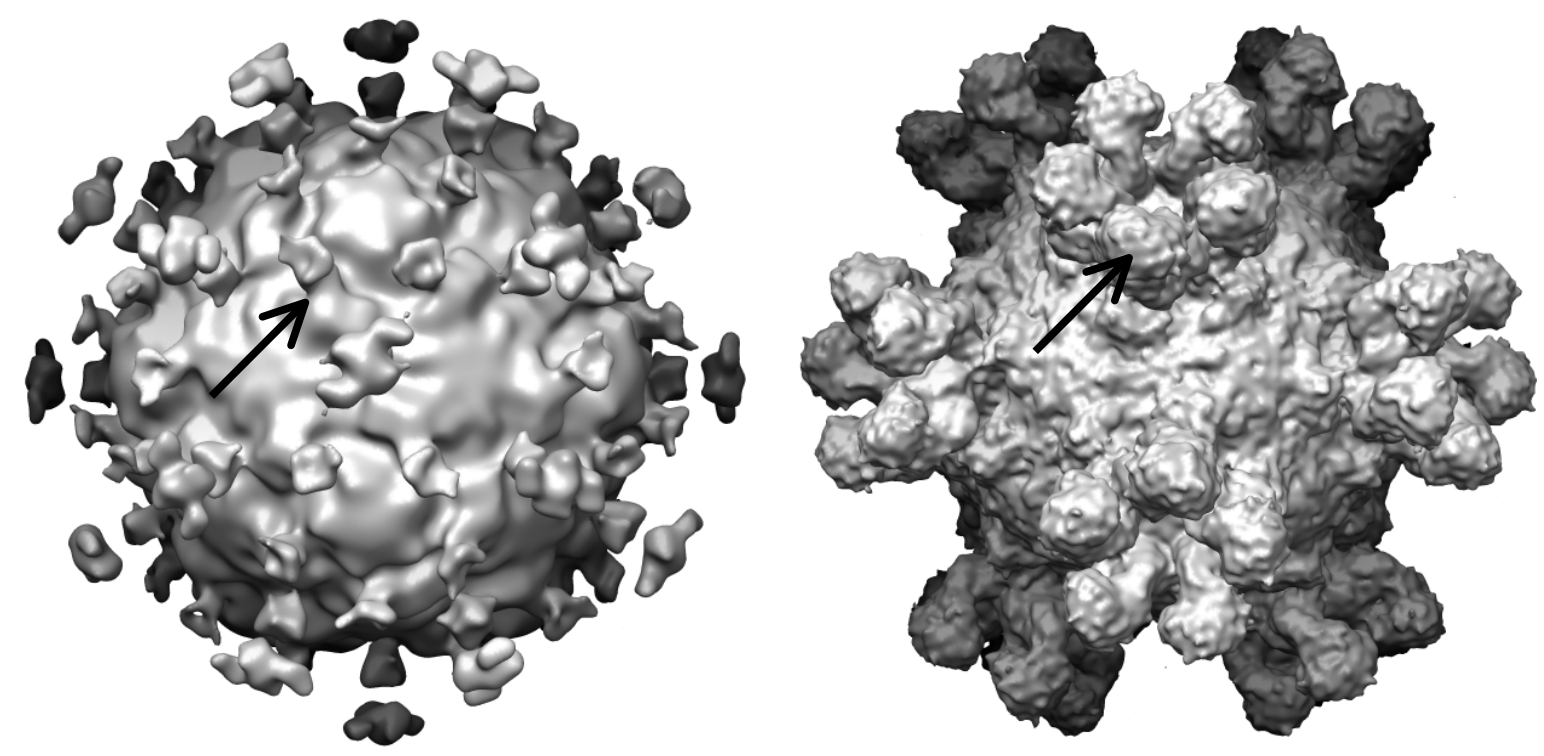

FIG. 1. Surface renderings of reconstructions of poliovirus-Fab complexes. Left, poliovirus 80S particle with a Fab to the first 31 amino acids of VP1 protein. An arrow shows where residues 31-32 were predicted to be in the Bubeck et al. model [3]. The Fab connects to the virus at this point. Right, poliovirus $135 \mathrm{~S}$ particle with a Fab to the region designated as the pivot point in the Belnap et al. [2] and Bubeck et al. [3] models. Five complete Fab molecules surround each fivefold vertex of the virus particle. The Fab density on the right contains the definition of a full Fab molecule (see arrow). Conversely, on the left, the Fab density is incomplete. This is most likely due to the mobility of residues $1-31$, so that during icosahedral averaging (to compute the $3 \mathrm{D}$ reconstruction the densities were smeared. The two panels are not to scale. 